\title{
Nutricionistas egressos da Universidade Federal de \\ Santa Catarina: áreas de atuação, distribuição \\ geográfica, índices de pós-graduação e de filiação aos órgãos de classe
}

\section{Nutrition graduates from Universidade Federal de \\ Santa Catarina: areas of performance, geographical \\ distribution, indexes of postgraduate degree and of affiliation to class associations}

Emilaura ALVES 1

Camila Elizandra ROSSI ${ }^{1}$

Francisco de Assis Guedes de VASCONCELOS²

\section{R E S U M O}

\section{Objetivo}

Esta investigação teve como objetivo avaliar o perfil dos nutricionistas egressos da Universidade Federal de Santa Catarina, no período de 1983 a 2000, buscando identificar as áreas e cidades de atuação profissional e os índices de realização de Pós-Graduação, bem como observar a filiação às entidades de classe.

\section{Métodos}

A metodologia consistiu na aplicação de um questionário auto-resposta, previamente testado, o qual foi remetido aos egressos via Correios, endereços eletrônicos e fax. Para análise dos dados, foi utilizado o programa Microsoft Access.

\footnotetext{
${ }^{1}$ Curso de Graduação em N utrição, Universidade Federal de Santa Catarina. Bolsistas de Iniciação Científica do $\mathrm{PIBIC/CNPq/BIP/UFSC.}$

2 Departamento de Nutrição, Centro de Ciências da Saúde, Universidade Federal de Santa Catarina. Campus Universitário, Trindade, 88040-900, Florianópolis, SC, Brasil. Correspondência para/Correspondence to: F.A.G. VASCONCELOS. E-mail: fguedes@floripa.com.br
} 


\section{Resultados}

As áreas de atuação que mais absorvem este recurso profissional são Alimentação Coletiva (28,9\% ), Nutrição Clínica $(27,0 \%)$, Ensino $(20,7 \%)$, Saúde Pública $(12,6 \%)$ e Outras (10,8\%). Evidencia-se que $51,4 \%$ dos nutricionistas atuam no município de Florianópolis, 8,1\% em Balneário Camboriú e 8,1\% em Blumenau. A maioria dos egressos está filiada aos Conselhos Regionais de Nutricionistas e um grande contingente $(84,7 \%)$ ao Conselho Regional de Nutricionistas da segunda região, mas vinculam-se à Associação Catarinense de Nutrição apenas $27,5 \%$ dos egressos.

\section{Conclusão}

Esta investigação vem confirmar a migração profissional entre as áreas de atuação do nutricionista prevista em estudos anteriores.

Termos de indexação: nutricionistas, egressos, perfil profissional do nutricionista.

\section{A B S T R A C T}

\section{Objective}

The objective of this investigation was to evaluate the profile of nutrition graduates from Federal University of Santa Catarina, in Brazil, in the period from 1983 to 2000, aiming to identify the areas and cities of professional performance and the indexes of accomplishment of postgraduate degrees, as well as to observe the affiliation to the class associations.

\section{Methods}

The methodology consisted of the application of a self-answer questionnaire, previously tested, which was sent to the nutritionists through mail, electronic addresses and fax. For data analysis, the Microsoft Access program was used.

\section{Results}

The areas of performance that absorb most of this professional resource are Collective Feeding (28.9\%), Clinical Nutrition $(27.0 \%)$, Teaching $(20.7 \%)$, Public Health $(12.6 \%)$ and Others $(10.8 \%)$. It was found that $51.4 \%$ of the nutritionists work in the city of Florianópolis, $8.1 \%$ in Balneário Camboriú and $8.1 \%$ in Blumenau. The great majority of graduates are associated with the Regional Council of Nutritionists and a great contingent (84.7\%) with the Regional Council of Nutritionists of the second region, but only $27.5 \%$ of the nutritionists have joined the Santa Catarina Nutrition Association.

\section{Conclusion}

This investigation confirmed the professional migration among the areas of performance foreseen in previous studies.

Index terms: nutritionists, graduates, nutritionist's professional profile.

\section{N T R O D U Çã O}

Na década de 1940, ao emergir o nutricionista no Brasil, o campo de trabalho deste profissional conformou-se dentro de duas grandes áreas de conhecimento e/ou especialização: a
Nutrição Clínica (Dietoterapia) e a Alimentação Institucional (Alimentação Coletiva). A área de Nutrição Clínica, caracterizada pela realização de ações de caráter individual e curativo, centradas na utilização do alimento (dieta) como agente de tratamento, desenvolveu-se inicialmente nos 
hospitais públicos e privados dos principais centros urbanos do país. Por sua vez, a área de Alimentação Coletiva, caracterizada particularmente pela realização de ações de administração, no sentido de racionalização da alimentação de coletividades sadias e enfermas, desenvolveu-se principalmente nos restaurantes do Serviço de Alimentação da Previdência Social (SAPS) e de empresas (indústrias) privadas, emergentes naquele contexto ${ }^{1-4}$.

$\mathrm{Na}$ década de 1960, surgiu a área de Nutrição em Saúde Pública (Nutrição Social) e consolidou-se a atuação do profissional em Ensino (Docência). A área de Nutrição em Saúde Pública, caracterizada particularmente pela realização de ações de caráter coletivo e preventivo, visando contribuir para que a produção, a distribuição e o consumo de alimentos sejam adequados e acessíveis a todos os indivíduos da comunidade, desenvolveu-se principalmente em instituições públicas, vinculadas aos setores saúde, educação, assistência social e desenvolvimento comunitário. A área de Ensino, caracterizada pela realização de atividades de ensino, pesquisa e extensão em Nutrição, desenvolveu-se inicialmente nos primeiros Cursos de Graduação em Nutrição implantados no país s.5. $^{3,5}$.

Nas últimas três décadas do século $X X$, verificou-se um intenso processo de expansão do número de Cursos de Graduação em Nutrição e conseqüentemente do número de nutricionistas no país ${ }^{(3)}$, o que tem propiciado tanto a ampliação e diversificação do mercado de trabalho como sensíveis alterações nas condições de trabalho do nutricionista brasileiro. Diante desta ampliação e diversificação dos campos de trabalho ocupados pelo nutricionista, o Conselho Federal de Nutricionistas (CFN) e a Associação Brasileira de Nutrição (ASBRAN) firmaram convênio para concessão do título de especialista em seis áreas de atuação/conhecimento profissional: Nutrição
Clínica, Alimentação Coletiva, Saúde Coletiva, Nutrição e Dietética, Ciência e Tecnologia de Alimentos e Educação $0^{7,8}$.

Em Santa Catarina, o Curso de Graduação em Nutrição da Universidade Federal (UFSC), o primeiro do Estado, foi criado em 10 de agosto de 1979 e implantado no primeiro semestre de 1980. Até junho de 2001, 18 turmas haviam colado grau, totalizando 375 nutricionistas egressos da UFSC.

Em relação aos campos de trabalho do nutricionista, estudo realizado em Florianópolis, SC, demonstrou que a grande maioria dos profissionais (84,4\% ) encontrava-se em instituições públicas, contra $15,6 \%$ atuando em instituições privadas e de economia mista. Em relação às áreas de atuação, o setor hospitalar absorvia 48,4\% dos nutricionistas (25,0\% em Nutrição Clínica e 23,4\% na Administração de Serviços de Nutrição e Dietética); a área de Alimentação Coletiva absorvia $18,7 \%$; a docência, $17,2 \%$; a Nutrição em Saúde Pública, 10,9\% e 4,8\% atuavam em outras áreas ${ }^{9}$.

Diante da consolidação e do avanço da profissão nos últimos 20 anos, estudos têm sido realizados visando retratar aspectos relacionados às áreas de atuação e condições de trabalho do nutricionista, sob diferentes perspectivas ou

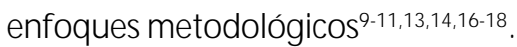

Este artigo se refere a parte dos resultados de uma pesquisa transversal descritiva que visou traçar um perfil da atuação profissional do universo de egressos do Curso de Graduação em Nutrição da UFSC. Desta forma, tem por objetivo descrever aspectos relacionados à atuação profissional (áreas de atuação/conhecimento e distribuição geográfica), à realização de cursos de Pós-Graduação e à filiação às entidades de classe, avaliando os nutricionistas formados pela UFSC, no período de dezembro de 1983 a fevereiro de 2000.

\footnotetext{
3 Em 9 de agosto de 2001, de acordo com dados do Consel ho Federal de Nutricionistas, o número de cursos em funcionamento passou a ser 132, com uma oferta de 10696 vagas anuais. Por sua vez, dados recentes apontam um efetivo de 29.188 nutricionistas registrados, até 30 de junho de 2001, nos distintos conselhos regionais (Conselho, 2001a) ${ }^{6}$.
} 


\section{CASUÍSTICA E MÉTODOS}

Os procedimentos metodológicos iniciais centraram-se no levantamento dos endereços residenciais e telefônicos do universo de 343 nutricionistas formados pela UFSC, ao longo do período investigado. Inicialmente recorreu-se às listagens de endereços dos profissionais cadastrados no Conselho Regional de Nutricionistas (CRN-2), na Associação Catarinense de Nutrição (ACAN) e nas malas-diretas de eventos organizados pelo Departamento de Nutrição da UFSC. No sentido de propiciar um maior número de endereços atualizados, outras estratégias também foram utilizadas, tais como o uso das Listas Telefônicas (Relação de Assinantes fornecida pela Telecomunicações de Santa Catarina S.A.) e consultas a colegas de turma já localizados. Ao final de tais procedimentos foram identificados os endereços de $296(86,3 \%)$ do total de nutricionistas egressos da UFSC.

Os dados foram coletados a partir da aplicação de um questionário auto-resposta, desenhado especificamente para atender os objetivos da pesquisa. A principal via de remessa do questionário foi o serviço de Correios. Outras vias de remessa tais como endereço eletrônico (e-mail), fax e entrega pessoal também foram utilizadas, em menor escala, quando a primeira e principal via (Correios) não funcionou. Retornaram respondidos 131 do total de questionários remetidos, perfazendo um índice de $44,2 \%$ de adesão à pesquisa.

A pós análise crítica de cada questionário respondido, as informações foram processadas no M icrosoft Access 97, o qual possibilitou a criação de um banco de dados. A partir dos relatórios criados no próprio programa, construíram-se gráficos e tabelas contendo a distribuição de freqüência das variáveis estudadas, acompanhadas de cálculos de medidas de tendência central e dispersão (médias e desvios-padrão), quando pertinentes.

Neste artigo, apresentam-se os resultados obtidos a partir da análise das seguintes variáveis: áreas de atuação profissional, distribuição geográfica, realização de cursos de Pós-Graduação e filiação às entidades representativas da categoria.

Em relação à área de atuação profissional, considerou-se apenas aquela correspondente ao emprego/atividade principal (aquele com maior carga horária de trabalho), uma vez que 32,4\% dos nutricionistas investigados possuíam mais de um emprego. Adotou-se este mesmo critério para a variável distribuição geográfica dos egressos segundo municípios de atuação profissional.

Quanto à realização de Pós-Graduação, observaram-se os seguintes critérios: consideraram-se somente os egressos com pós-graduação lato sensu ou stricto sensu na área de Nutrição e áreas afins; para os egressos que realizaram mais de um curso, considerou-se somente o de maior título, sendo cada profissional classificado em apenas uma modalidade de pós-graduação. Determinou-se o tempo para ingresso na primeira Pós-Graduação, subtraindo-se 0 ano de início da primeira Pós-Graduação do ano de conclusão da Graduação.

Quanto à filiação às entidades de classe, analisou-se em duas categorias: a) Conselho Regional de Nutricionistas (entidade responsável pela fiscalização e normatização do exercício legal da profissão, cujo vínculo tem caráter obrigatório), e b) Associações Científicas (entidades de caráter técnico-científico e cultural, cujo vínculo tem caráter facultativo).

RESULTADOSE DISCUSSÃO

\section{Áreas de atuação profissional}

Dos 131 nutricionistas que aderiram à pesquisa, 111 exerciam a profissão, correspondendo a $84,7 \%$ do total. Os demais, 20 egressos, relataram ter abandonado a profissão (exerceram a profissão por um determinado período e depois abandonaram), estar desempregado ou nunca ter 
exercido a profissão. Entre os profissionais em exercício, as áreas de atuação mais prevalentes foram: Alimentação Coletiva $(28,9 \%)$, Nutrição Clínica $(27 \%)$, Ensino $(20,7)$ e Saúde Pública $(12,6 \%$ ) (Tabela 1). Os demais $(10,8 \%)$ realizam atividades em áreas surgidas mais recentemente, tais como Assessoria, Consultoria, Nutrição Esportiva, Análise Sensorial e Controle da Qualidade de Alimentos, Representante Hospitalar, M arketing, Pesquisa, Administração de Supermercados, Gestão de Microempresa.

Observa-se, portanto, importante diferença entre estes resultados e aqueles obtidos por Vasconcelos (1991) ${ }^{9}$ em Florianópolis, onde 48,4\% dos nutricionistas atuavam em Hospitais e 18,7\% em Administração de Serviços de Alimentação de Empresas. No Rio de Janeiro, Prado \& Abreu $(1991)^{13}$ também encontraram dados semelhantes aos de Vasconcelos, com 51,3\% destes profissionais alocados em Hospitais e 22,4\% em empresas prestadoras de Serviços de Alimentação e Nutrição.

Nesta pesquisa pode-se observar o mesmo indicativo de migração profissional entre as áreas de atuação do nutricionista já sugerido por Gambardella et al. (2000)18. Quando estudou o perfil profissional dos nutricionistas egressos da Universidade de São Paulo (USP), Gambardella observou proporções próximas entre aqueles que trabalhavam em Nutrição Clínica $(36,6 \%$ ) e em Unidades de Alimentação e Nutrição (UAN) (31\% ), indicando uma transição da Nutrição Clínica para as UAN ou para qualquer outra área.

0 contingente de egressos atuantes em Ensino $(20,7 \%)$ encontrado nesta pesquisa surpreende quando comparado com os achados por Boog et al. (1989)11, 6,3\% , e por Gambardella et al. $(2000)^{18}, 1,4 \%$. Algumas hipóteses podem ser levantadas para explicar tais achados. Uma delas pode ter sido a facilidade de contato com os egressos que são docentes da UFSC, favorecendo a adesão total destes profissionais à pesquisa. Outra pode ter sido a criação do Curso de Graduação em Nutrição da Universidade do Vale de Itajaí (UNIVALI), em 1996, localizado em Itajaí, município próximo de Florianópolis, o qual contribuiu para uma maior absorção do nutricionista na área de ensino.

Tabela 1. Distribuição dos nutricionistas egressos da UFSC conforme a área de atuação Florianópolis, 2001.

\begin{tabular}{lcc}
\hline Área de atuação & Egressos (n) & Percentual (\%) \\
\hline Alimentação Coletiva & 32 & 28,9 \\
Nutrição Clínica & 30 & 27,0 \\
Ensino & 23 & 20,7 \\
Saúde Pública & 14 & 12,6 \\
Outras & & 1,8 \\
Assessoria & 2 & 1,8 \\
Gestão de Microempresa & 2 & 0,9 \\
Consultoria & 1 & 0,9 \\
Nutrição Esportiva & 1 & 0,9 \\
Análise Sensorial e Controle de Qualidade de Alimentos & 1 & 0,9 \\
Representante Hospitalar & 1 & 0,9 \\
Marketing & 1 & 0,9 \\
Pesquisa & 1 & 0,9 \\
Administração de Supermercados & 1 & 0,9 \\
Sem informação & 1 & 100,0 \\
\hline Total & 111 & \\
\hline
\end{tabular}


O pequeno número de profissionais atuando na área de Nutrição em Saúde Pública $(12,6 \%)$ foi também constatado por Boog et al. $(1989)^{11}$, por Vasconcelos $(1991)^{14}$ e por Gambardella et al. $(2000)^{18}$, os quais encontraram as seguintes proporções, respectivamente: $6,3 \%$, $10,9 \%$ e $7 \%$.

\section{Distribuição geográfica dos egressos}

De acordo com os resultados desta pesquisa, 57 egressos $(51,4 \%$ do total de nutricionistas empregados) atuam no município de Florianópolis (Tabela 2). Em seguida, destacam-se as cidades de Balneário Camboriú e Blumenau, ambos com 8,1\%, São José com 5,4\% e joinville com $4,5 \%$ do total destes profissionais. As demais cidades do Estado apresentam um exíguo contingente destes nutricionistas. Em outros Estados brasileiros, como São Paulo e Minas Gerais, localizaram-se seis egressos. Localizou-se um dos egressos no exterior (Alemanha).

Em estudo realizado por Vasconcelos $(1991)^{9}$ já se observava proporção semelhante deste profissional concentrado na capital catarinense, correspondendo a $54,7 \%$ do total do Estado. Segundo Vasconcelos (1991) ${ }^{9}$, isto se justifica por Florianópolis "ser o principal centro político e concentrar as principais empresas e instituições estatais", ainda que não constitua 0 "principal centro econômico nem populacional" do Estado. A proporção de nutricionistas em outras cidades de Santa Catarina, reconhecidas por sua importância econômica, durante dez anos não sofreu grandes mudanças. Vasconcelos identificou uma concentração de $8,3 \%$ dos nutricionistas

Tabela 2. Distribuição dos nutricionistas egressos da UFSC segundo a cidade onde exercem a profissão Florianópolis, 2001.

\begin{tabular}{|c|c|c|}
\hline Cidade & Egressos (n) & Percentual (\%) \\
\hline Florianópolis & 57 & 51,4 \\
\hline Balneário Camboriú & 9 & 8,1 \\
\hline Blumenau & 9 & 8,1 \\
\hline São José & 6 & 5,4 \\
\hline Joinville & 5 & 4,5 \\
\hline Lages & 3 & 2,7 \\
\hline Tubarão & 3 & 2,7 \\
\hline Chapecó & 2 & 1,8 \\
\hline Xanxerê & 2 & 1,8 \\
\hline São Joaquim & 1 & 0,9 \\
\hline Ibirama & 1 & 0,9 \\
\hline Indaial & 1 & 0,9 \\
\hline Içara & 1 & 0,9 \\
\hline Videira & 1 & 0,9 \\
\hline Criciúma & 1 & 0,9 \\
\hline Caçador & 1 & 0,9 \\
\hline \multicolumn{3}{|l|}{ Fora do Estado } \\
\hline São Paulo & 3 & 2,7 \\
\hline Piracicaba & 1 & 0,9 \\
\hline Ouro Preto & 1 & 0,9 \\
\hline Uberlândia & 1 & 0,9 \\
\hline \multicolumn{3}{|l|}{ Outro país } \\
\hline Alemanha & 1 & 0,9 \\
\hline Sem informação & 1 & 0,9 \\
\hline Total & 111 & 100,0 \\
\hline
\end{tabular}


do Estado em Blumenau, 7,8\% em Joinville, $3,7 \%$ em Criciúma e 2,6\% em Chapecó. Comparando-se com os resultados desta pesquisa, a proporção mais desigual pertence à cidade de Criciúma. Os demais valores referenciados guardam semelhanças com aqueles obtidos nesta investigação.

\section{Índices de realização de cursos de Pós-Graduação}

Neste estudo, $61,8 \%$ dos nutricionistas investigados cursaram ou estavam cursando alguma modalidade de Pós-Graduação na área de Nutrição e em outras áreas afins. Os demais, $38,2 \%$, possuem apenas a graduação.

Encontraram-se nutricionistas com título de especialista $(35,8 \%)$, mestre $(16,8 \%)$ e doutor $(9,2 \%$ ) (Tabela 3 ). Os resultados aqui obtidos superaram os índices de realização de Pós-Graduação por estes profissionais em nível nacional. De acordo com pesquisa realizada pelo
CFN, $24,0 \%$ dos nutricionistas realizaram curso de Pós-Graduação, dos quais 2,0\% são mestres e $0,5 \%$ são doutores ${ }^{19}$.

Dentre os cursos de Pós-Graduação realizados pelos nutricionistas, destacam-se como os mais cursados: especialização em Terapia Nutricional e especialização em Gerência da Qualidade na Produção de Refeições Coletivas, mestrado em Ciência dos Alimentos e doutorado em Engenharia de Produção. Ressalta-se que estes cursos são oferecidos pela UFSC, justificando de certa forma, uma maior procura por parte destes profissionais e uma maior facilidade para ingressarem nos mesmos.

Em relação ao tempo que estes nutricionistas levam para ingressar na primeira Pós-Graduação, entre os 81 pós-graduados observou-se uma média de 4,7 $\pm 3,7$ anos entre o início da primeira Pós-Graduação e o término da Graduação, com uma amplitude localizada entre zero e 12 anos.

Tabela 3. Distribuição dos nutricionistas egressos da UFSC de acordo com modalidade de Pós-Graduação realizada Florianópolis, 2001.

\begin{tabular}{lcc}
\hline Modalidades de Pós-Graduação & Egressos (n) & Percentual (\%) \\
\hline Especialização & 47 & 35,8 \\
Mestrado & 22 & 16,8 \\
Doutorado & 12 & 9,2 \\
Apenas graduação & 50 & 38,2 \\
\hline Total & 131 & 100,0 \\
\hline
\end{tabular}

Tabela 4. Distribuição dos nutricionistas egressos da UFSC conforme filiação ao Conselho Regional de Nutricionista Florianópolis, 2001.

\begin{tabular}{|c|c|c|c|}
\hline Conselhos Regionais de Nutrição & Egressos (n) & Percentual (\%) & Percentual acumulado (\%) \\
\hline CRN - 1 & 1 & 0,8 & 0,8 \\
\hline CRN - 2 & 111 & 84,7 & 85,5 \\
\hline CRN - 3 & 3 & 2,3 & 87,8 \\
\hline CRN - 4 & 2 & 1,5 & 89,3 \\
\hline Nenhuma & 13 & 9,9 & 99,2 \\
\hline Sem informação & 1 & 0,8 & 100,0 \\
\hline Total & 131 & 100,0 & - \\
\hline
\end{tabular}

Nota: $C R N=$ Conselho Regional de Nutrição 


\section{Filiação às entidades representativas da categoria}

A maioria está vinculada ao Conselho Regional de Nutricionistas da segunda região (CRN-2), do qual faz parte o estado de Santa Catarina (Tabela 4). Uma pequena parcela de nutricionistas exerce a profissão em outras regiões do Brasil e, portanto, estão vinculados a outros conselhos, como CRN-1 (Goiás, Mato Grosso e Tocantins), CRN-3 (São Paulo, Paraná e Mato Grosso do Sul) e CRN-4 (Rio de Janeiro, Espírito Santo e M inas Gerais).

Foram identificados 39 profissionais filiados às associações científicas, entre elas a Associação Catarinense de Nutrição (ACAN), a Sociedade Brasileira de Alimentação e Nutrição (SBAN) e a Sociedade Brasileira de Nutrição Parenteral e Enteral (SBNPE) (Tabela 5). Destaca-se o número de egressos vinculados à ACAN (27,5\% do total), considerado baixo, uma vez que a maioria dos egressos exerce a profissão no Estado e poderia estar associada a esta entidade científica, contribuindo para o fortalecimento da categoria profissional. Resultado semelhante pode ser observado em estudo realizado por Boog et al. $(1989)^{11}$, onde apenas $22,8 \%$ da amostra analisada estava filiada à Associação Paulista de Nutrição (APAN).

\section{O N C L U S Ã O}

Este estudo da prática profissional dos nutricionistas egressos da UFSC possibilita traçar as seguintes constatações finais:
1. Considerando os egressos que estavam em exercício da profissão, o percentual de nutricionistas trabalhando com Nutrição Clínica e Alimentação Coletiva é muito semelhante. A diferença entre os percentuais de profissionais atuantes nestas duas áreas diminuiu em relação ao início da década passada, divergindo dos estudos anteriores. As áreas de atuação que menos concentravam profissionais provavelmente cresceram e diversas outras surgiram, acarretando a permuta de nutricionistas entre as áreas consideradas clássicas e as recém-surgidas. Sendo assim, sugere-se a realização de novos estudos para investigação da oferta de emprego e dos níveis salariais por área de atuação e para verificação das possíveis associações entre estas variáveis.

2. A distribuição geográfica dos egressos, de acordo com as cidades onde exercem as atividades profissionais, concentra-se na capital catarinense, o principal centro político do Estado. A segunda área do Estado com mercado de trabalho atrativo para este profissional compreende as regiões com forte pólo industrial, como Blumenau, São José e Joinville. Balneário Camboriú constitui-se na segunda cidade com a maior concentração de profissionais, devido à implantação, em 1996, do Curso de Graduação em Nutrição da Universidade do Vale do Itajaí (UNIVALI), o qual atraiu parte dos egressos da UFSC na qualidade de docentes.

3. A filiação dos nutricionistas aos Conselhos Regionais apresenta-se bastante expressiva, em parte devido à obrigatoriedade da

Tabela 5. Distribuição dos nutricionistas egressos da UFSC conforme filiação às associações científicas Florianópolis, 2001.

\begin{tabular}{lccc}
\hline Associações Científicas & Egressos (n) & Percentual (\%) & Percentual acumulado (\%) \\
\hline ACAN & 36 & 27,5 & 27,5 \\
SBAN & 2 & 1,5 & 29,0 \\
SBNPE & 1 & 0,8 & 29,8 \\
Nenhuma & 91 & 69,4 & 99,2 \\
Não obtido & 1 & 0,8 & 100,0 \\
\hline Total & 131 & 100,0 & - \\
\hline
\end{tabular}

Nota: ASCAN = Associação Catarinense de Nutrição; SBNPE = Sociedade Brasileira de Alimentação e Nutrição; SBNPE = Sociedade Brasileira de Nutrição Parenteral e Enteral. 
inscrição para o exercício legal da profissão. 0 percentual de egressos vinculados ao CRN-2 demonstra-se majoritário porque a maioria exerce a profissão em Santa Catarina. Já o vínculo com a asso ciação científica (ACAN) apresenta-se bem menor. A filiação dos egressos a estas entidades reflete o grau primário de mobilização e organização da categoria profissional no Estado. Estas entidades estão comprometidas com a representação da categoria profissional nas esferas políticas, técnico-científicas e administrativas, conforme o cunho social. Portanto, faz-se necessário que os nutricionistas assumam um comprometimento maior com as associações científicas e com o Conselho Regional.

4. Entre os 131 egressos que participaram da pesquisa, observa-se um índice elevado de realização de cursos de Pós-Graduação, tanto lato sensu quanto stricto sensu, superando o índice nacional dessa categoria profissional. Entre as modalidades cursadas, encontra-se um número expressivo de especializações, as quais provavelmente advêm das exigências da prática profissional cotidiana. Os índices de realização de mestrado e doutorado são também expressivose talvez estejam correlacionados com a ampliação desse mercado de trabalho e conseqüente aumento do interesse pela área de Ensino. No entanto, 0 ingresso nos cursos de Pós-Graduação não representa, de imediato, 0 destino profissional destes nutricionistas, visto que levam alguns anos para iniciarem a primeira Pós-Graduação.

Estudos visando caracterizar o perfil profissional dos nutricionistas merecem maior atenção pelo conjunto de pesquisadores da área, uma vez que a categoria profissional conquista novos e diversificados mercados de trabalho, onde as tendências e exigências profissionais encontram-se em constante transformação.

\section{REFERÊ NCIAS}

1. Associação Brasileira de Nutrição. Histórico do Nutricionista no Brasil - 1939 a 1989: coletânea de depoimentos e documentos. São Paulo: Ateneu; 1991.
2. L'A bbate, S. As Políticas de Alimentação e Nutrição no Brasil. I. Período de 1940 a 1964. Rev Nutr PUCCAMP 1988; 1(2):87-138.

3. Ypiranga L, GIL MF. Formação profissional do nutricionista: Por que mudar? In: CUNHA DTO, Ypiranga L, Gil MF, organizadores. II Seminário nacional sobre o ensino de Nutrição. Goiânia: FEBRAN; 1989.

4. Vasconcelos FAG. Os Arquivos Brasileiros de Nutrição: uma revisão sobre produção científica em Nutrição no Brasil (1944 a 1968). Cad Saúde Pública 1999; 15(2):303-16.

5. Vasconcelos FAG. Fome, eugenia e constituição do campo da nutrição em Pernambuco: uma análise de Gilberto Freyre, Josué de Castro e Nelson Chaves. História, Ciências, Saúde - Manguinhos 2001; 8(2):315-39.

6. Conselho Federal de Nutricionistas. Quadro estatístico do 2o trimestre/2001 (1\%/04/2001 a 30/06/2001) [online] 2001a [capturado 21 nov 2001]. Disponível em: http://www.cfn.org.br/ variavel/destaque/quadro_estatístico6.htm

7. Conselho Federal de Nutricionistas. 31 de agosto: dia do Nutricionista. Rev CFN 2001b; 2(4):9-12.

8. Conselho Federal de Nutricionistas. Edital define diretrizes para a concessão do título de especialista. Rev CFN 2001c; 2(5):5.

9. Vasconcelos FAG. Um perfil do nutricionista em Florianópolis - Santa Catarina. Rev Ciênc Saúd 1991; 10(1/2):73-86.

10. Boog MCF, Rodrigues KRM, Silva SMF. Situação profissional dos nutricionistas egressos da PUCCAMP I. Áreas de atuação, estabilidade, abandono da profissão, desemprego. Rev Nutr 1998; 11(2):139-52.

11. Boog MCF, Rodrigues KRM, Silva SMF. Situação profissional dos nutricionistas egressos da PUCCAMP II. Relações hierárquicas, atuação profissional, avaliação da formação recebida, percepção de si como profissional da saúde, atualização e filiação a entidades profissionais. Rev Nutr PUCCAM P 1989; 2(1):55-87. 
12. Bosi MLM. Profissionalização e conhecimento: a nutrição em questão. São Paulo: Hucitec; 1996.

13. Prado SD, Abreu MSD. Nutricionista: onde trabalha? Quais suas condições de trabalho? Rev Nutr PUCCAMP 1991; 4(1/2):65-91.

14. Rotemberg S, Prado SD. Nutricionistas: quem somos? Rev Nutr PUCCAMP 1991; 4(1/2):40-64.

15. Prado SD. Autonomia: tentadora e reificada. Estudo sobre os nutricionistas dos centros municipais de saúde do Rio de Janeiro [dissertação]. Escola Nacional de Saúde Pública, Fundação Oswaldo Cruz; 1993.

16. Costa NM SC. Repensando a formação acadêmica e a atuação profissional do nutricionista: um estudo com egressos da Universidade Federal de
Goiás (UFG). Rev Nutr PUCCAMP 1996; $9(2): 154-77$.

17. Viana SV. Nutrição, trabalho e sociedade. São Paulo: Hucitec; 1996.

18. Gambardella AMD, Ferreira CF, Frutoso MFP. Situação profissional de egressos de um curso de Nutrição. Rev Nutr 2000; 13(1):37-40.

19. Nutrinews. 31 de agosto Dia do Nutricionista: uma categoria em plena transformação [online] 2001 [jun 2001]. Disponível em: http://nutrinew s.com.br/ edicoes/9909/mat02.html

Recebido para publicação em 7 de março e aceito em 18 de setembro de 2002 